\title{
A hybrid method for vibroacoustic coupling
}

\section{Application to the noise radiated around an engine compartment}

\author{
Emeline Sadoulet-Reboul* — Alain Le Bot** \\ Joël Perret-Liaudet** \\ * Institut FEMTO-ST UMR CNRS 6174 \\ Département de Mécanique Appliquée \\ Université de Franche-Comté \\ 24 chemin de l'épitaphe F-25000 Besançon \\ Emeline.Sadoulet-Reboul@univ-fcomte.fr \\ ** Laboratoire de Tribologie et Dynamique des Systèmes \\ UMR CNRS 5513 \\ Ecole Centrale de Lyon \\ 54 avenue Guy de Collongue F-69134 Ecully cedex \\ \{Alain.Le-Bot,Joel.Perret-Liaudet\}@ec-lyon.fr
}

\begin{abstract}
A hybrid method is proposed to study the noise radiated in the high frequency range by a gearbox inside an engine compartment. Due to its elastodynamic characteristics, the vibratory behaviour of the transmission lies in the low frequency range. However, the noise radiated is rather in the high frequency range facing the size of the acoustical cavity. Thus the proposed hybrid method allows to couple a low-frequency vibratory approach to the radiative transfer method used for the high frequency acoustical calculation. The coupling is realized through equivalent energy sources introduced on the surface of the vibrating structure.

RÉSUMÉ. Cette étude présente l'application d'une méthode hybride pour étudier le rayonnement acoustique d'une boîte de vitesses placée au sein d'un encapsulage moteur. Au regard des caractéristiques élastodynamiques de la transmission, son comportement vibratoire se situe en basses fréquences. En revanche, au regard de la taille du compartiment où se propage le son, le bruit rayonné se situe davantage en hautes fréquences. C'est pourquoi une méthode hybride est proposée ici afin de coupler un calcul vibratoire basses fréquences à un calcul acoustique hautes fréquences réalisé à l'aide de la méthode du transfert radiatif. Pour réaliser ce couplage, des sources énergétiques équivalentes sont introduites à la surface de la structure vibrante.

KEYWORDS: vibroacoustics, mechanism, high frequency, equivalent sources.

MOTS-CLÉS : vibroacoustique, mécanisme, hautes fréquences, sources équivalentes.
\end{abstract}

DOI:10.3166/REMN.17.689-699 @ 2008 Lavoisier, Paris

REMN - 17/2008. Giens 2007, pages 689 to 699 


\section{Introduction}

The prediction of the noise radiated by a structure located inside an acoustical cavity is a complex problem. Indeed, in case only few vibratory modes of the structure are excited, its vibratory behaviour is rather in the low frequency range. Nevertheless, with regard to the size of the cavity where the structure radiates, the number of acoustical modes excited in the cavity is high enough so that the noise radiated lies in the high frequency range. Thus, a vibratory low-frequency study has to be coupled to an acoustical high-frequency one. This coupling gives rise to two main difficulties. First of all, structural low-frequency approaches lead to the parietal velocity field on the vibrating structure whereas acoustical high-frequency methods relie on energy quantities. Hence arise a problem of data compatibility. Besides, at high frequencies, wavelengths are small compared to the characteristic size of structures and numerical approaches based on a spatial discretization become time-consuming. Thus, the finite element method or the finite element method coupled to the boundary element method classically used to solve a vibroacoustic problem require all the finer meshes as the frequency increases and are not adapted to such a high frequency study.

A method called hybrid has been developed to realize this coupling. This method is applied in this paper to predict the noise radiated by a gearbox integrated to a power plant inside an engine compartment. With regard to the elastodynamic characteristics of a gearbox, its vibratory behaviour lies in the low frequency range so this study case is adapted to the hybrid approach. The velocity field on the surface of the housing is used as an entry data for the acoustical study carried out using the radiative transfer method. This method relies on analogies with heat transfer as the radiosity method well-known in room acoustics (Miles, 1984), (Kuttruff, 1997). The main point of the hybrid method is the introduction of equivalent sources to ensure the coupling as described in the first section of this paper. Then, the radiative transfer method is applied to compute the radiated acoustical field. The final section is dedicated to the proposed application and results are compared in terms of precision and computational time to those given by the boundary element method.

\section{Hybrid coupling with equivalent sources}

Firstly, the vibrating structure is considered as if radiating in the free-field, that is to say without its acoustical environment. The hybrid method is based on the introduction of equivalent sources on the structure surface. To compute the amplitude of these sources, an analogy is established between the energy density deduced from the Kirchhoff-Helmholtz equation and the formulation as used in the radiative transfer method (Reboul, 2005), (Sadoulet-Reboul et al., 2007). 


\subsection{Derivation of the equivalent sources}

The sound pressure field $p$ at point $\mathbf{r}$ in the acoustical domain is given by the Kirchhoff-Helmholtz integral equation which relates this pressure field to the sound pressure and to the normal acceleration $\gamma_{n}$ at point q on the surface $S$ of the vibrating structure (Lesueur, 1988),

$$
p(\mathbf{r})=\int_{S} \rho \gamma_{n}(\mathbf{q}) g(\mathbf{q}, \mathbf{r})+p(\mathbf{q}) \frac{\partial g}{\partial n}(\mathbf{q}, \mathbf{r}) \mathrm{d} S
$$

$\rho$ denotes the fluid density, $g(\mathbf{q}, \mathbf{r})=e^{-\mathrm{j} k r} / 4 \pi r$ is the Green's function in the free field, $k=k_{0}-\mathrm{j} m / 2$ is the complex wavenumber where $k_{0}$ is the real part of the wavenumber and $m$ is the atmospheric attenuation factor, $r=|\mathbf{r}-\mathbf{q}|$ is the sourcereceiver distance between points $\mathbf{q}$ and $\mathbf{r}$, and $\partial / \partial n$ is the derivative in the direction of the outward normal $\mathbf{n}_{\mathbf{q}}$ at point $\mathbf{q}$. The acoustical energy density is related to the mean square of the pressure through the following equation,

$$
W(\mathbf{r})=\frac{|p(\mathbf{r})|^{2}}{2 \rho c^{2}} .
$$

$c$ denotes the speed of sound. Substituting Equation [1] in Equation [2], the energy density can be written as,

$$
\begin{aligned}
W(\mathbf{r})= & \iint_{S \times S} \frac{1}{2 \rho c^{2}} p(\mathbf{q}) p^{*}\left(\mathbf{q}^{\prime}\right) \frac{\partial g(\mathbf{q}, \mathbf{r})}{\partial n} \frac{\partial g^{*}\left(\mathbf{q}^{\prime}, \mathbf{r}\right)}{\partial n} \mathrm{~d} S^{\prime} \mathrm{d} S \\
& +\iint_{S \times S} \frac{\rho}{2 c^{2}} \gamma_{n}(\mathbf{q}) \gamma_{n}^{*}\left(\mathbf{q}^{\prime}\right) g(\mathbf{q}, \mathbf{r}) g^{*}\left(\mathbf{q}^{\prime}, \mathbf{r}\right) \mathrm{d} S^{\prime} \mathrm{d} S \\
& +\operatorname{Re}\left(\iint_{S \times S} \frac{1}{c^{2}} \gamma_{n}(\mathbf{q}) p^{*}\left(\mathbf{q}^{\prime}\right) g(\mathbf{q}, \mathbf{r}) \frac{\partial g^{*}\left(\mathbf{q}^{\prime}, \mathbf{r}\right)}{\partial n} \mathrm{~d} S^{\prime} \mathrm{d} S\right),
\end{aligned}
$$

where ${ }^{*}$ denotes the conjugate quantity. Equivalent sources are then derived using a far-field approximation and taking the ensemble average of Equation [3]. $<.>$ denotes the ensemble averaging operator. The difference of acoustical way between two source points is estimated in the far-field, or yet as if plane waves were emitted. It leads to formulations where the sources do not depend on the position of the receiver point $\mathbf{r}$ but only on the direction of emission $\mathbf{u}$ towards this point. Such formulations are adapted to a ray method as the radiative transfer method (Le Bot et al., 2000). Finally, equivalent sources $\sigma_{e}$ are divided into three contributions, $\sigma_{p p}, \sigma_{\gamma \gamma}$ and $\sigma_{\gamma p}$, respectively called pressure, velocity and intensity sources such that,

$$
\sigma_{e}=\sigma_{p p}+\sigma_{\gamma \gamma}+\sigma_{\gamma p} .
$$


They can be written as :

$$
\begin{aligned}
\sigma_{p p}(\mathbf{q}, \mathbf{u}) & =\operatorname{Re}\left(\frac{|k|^{2}}{8 \pi \rho c}\left(\mathbf{u} \cdot \mathbf{n}_{\mathbf{q}}\right)^{2} \int_{S} R_{p p}\left(\mathbf{q}, \mathbf{q}^{\prime}\right) e^{-\mathrm{j} k_{0} d \cos \Theta} \mathrm{d} S^{\prime}\right), \\
\sigma_{\gamma \gamma}(\mathbf{q}, \mathbf{u}) & =\operatorname{Re}\left(\frac{\rho}{8 \pi c} \int_{S} R_{\gamma \gamma}\left(\mathbf{q}, \mathbf{q}^{\prime}\right) e^{-\mathrm{j} k_{0} d \cos \Theta} \mathrm{d} S^{\prime}\right) \\
\sigma_{\gamma p}(\mathbf{q}, \mathbf{u}) & =\operatorname{Re}\left(\frac{\mathrm{j} k^{*}}{4 \pi c}\left(\mathbf{u} \cdot \mathbf{n}_{\mathbf{q}}\right) \int_{S} R_{\gamma p}\left(\mathbf{q}, \mathbf{q}^{\prime}\right) e^{-\mathrm{j} k_{0} d \cos \Theta} \mathrm{d} S^{\prime}\right) .
\end{aligned}
$$

As depicted on Figure 1, $\mathbf{u}$ is the unit vector from the source point $\mathbf{q}$ to the receiver point $\mathbf{r}, \mathbf{n}_{\mathbf{q}}$ is the unit outward normal at point $\mathbf{q}, d$ is the distance between two source points $\mathbf{q}$ and $\mathbf{q}^{\prime}, \Theta$ is the angle of emission from the source point q. $R_{p p}\left(\mathbf{q}, \mathbf{q}^{\prime}\right)=<p(\mathbf{q}) p^{*}\left(\mathbf{q}^{\prime}\right)>, R_{\gamma \gamma}\left(\mathbf{q}, \mathbf{q}^{\prime}\right)=<\gamma_{n}(\mathbf{q}) \gamma_{n}^{*}\left(\mathbf{q}^{\prime}\right)>$ et $R_{\gamma p}\left(\mathbf{q}, \mathbf{q}^{\prime}\right)=<\gamma_{n}(\mathbf{q}) p^{*}\left(\mathbf{q}^{\prime}\right)>$ are the pressure, acceleration and intensity crossspectral densities, that is the cross-correlation of two fields at points $\mathbf{q}$ and $\mathbf{q}^{\prime}$.

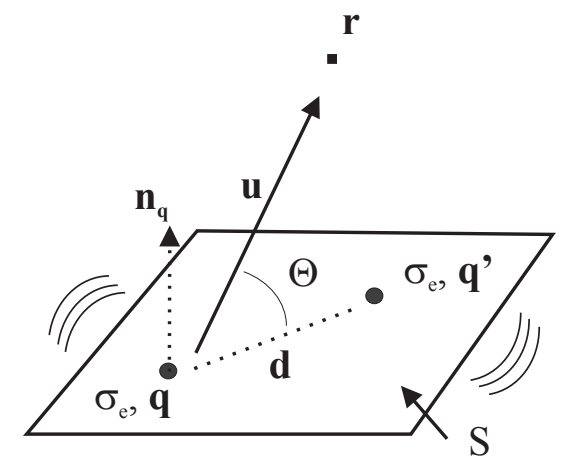

Figure 1. Equivalent sources are introduced at point $\mathbf{q}$ on the vibrating surface $S$. They emit in the direction $\mathbf{u}$ towards the receiver point $\mathbf{r} . d$ is the distance between sources at points $\mathbf{q}$ and $\mathbf{q}^{\prime}, \Theta$ is the angle of emission at point $\mathbf{q}$, and $\mathbf{n}_{\mathbf{q}}$ is the unit outward normal at point $\mathbf{q}$

Thus, the acoustical energy density is given by summing the contributions of the equivalent sources,

$$
W(\mathbf{r})=\int_{S} \sigma_{e}(\mathbf{q}, \mathbf{u}) G(\mathbf{q}, \mathbf{r}) \mathrm{d} S .
$$

$G(\mathbf{q}, \mathbf{r})=e^{-m r} / 4 \pi r^{2} c$ is the kernel function for energy density. The hybrid method carries on the FAQP (Frequency Averaged Quadratic Pressure), simplified BEM, and EBEA (Energy Boundary Element Analysis) methods (Guyader et al., 2000), (Kim et al., 2002), (Wang et al., 2004). 


\subsection{Numerical implementation}

The surface $S$ of the vibrating structure is divided into $n$ triangular or quadrangular elements. One equivalent source is introduced at the barycentre $\mathbf{q}_{\mathbf{i}}$ of each element $i$. The amplitude of each source denoted $\sigma_{e, i}$ is assumed to be constant on each element and depends on the direction of emission $\Theta$ from the source point $q_{i}$ to the receiver point $\mathbf{r}$. Surface integrals are computed using a Gaussian quadrature rule. For instance, velocity sources $\sigma_{\gamma \gamma}$ are written as :

$$
\begin{gathered}
\sigma_{\gamma \gamma, i}(\Theta)= \\
\operatorname{Re}\left(\frac{\rho}{8 \pi c} \sum_{i^{\prime}=1}^{n} \sum_{l=1}^{n_{i^{\prime}, x}} \sum_{m=1}^{n_{i^{\prime}, y}}\left|J_{l, m}\right| \quad W_{l} W_{m} R_{\gamma \gamma}\left(\mathbf{q}_{\mathbf{i}}, \mathbf{q}_{\mathbf{l}, \mathbf{m}}\right) e^{-\mathrm{j} k_{0} d_{l, m} \cos \Theta}\right) .
\end{gathered}
$$

$\left|J_{l, m}\right|$ and $\left(W_{l}, W_{m}\right)$ are respectively the Jacobian determinant and the weight at the integration point $\mathbf{q}_{\mathbf{l}, \mathbf{m}}$ on the $i^{\prime}$-th element, which is the integration element. $d_{l, m}=$ $\left|\mathbf{q}_{\mathbf{i}}-\mathbf{q}_{\mathbf{l}, \mathbf{m}}\right|$ is the distance between $\mathbf{q}_{\mathbf{i}}$ and $\mathbf{q}_{\mathbf{l}, \mathbf{m}} \cdot n_{i^{\prime}, x}$ and $n_{i^{\prime}, y}$ are the number of Gauss points along each dimension of the $i^{\prime}$-th element. Velocity and pressure fields on the mesh associated to the equivalent sources are deduced from the known fields on the structural mesh and the fields at the integration points are then computed using the shape function on each element.

\section{Acoustical field predicted from the radiative transfer method}

\subsection{Radiative transfer equations}

The equivalent sources $\sigma_{e}$ are introduced in the radiative transfer method, and fictitious sources $\sigma_{f}$ are added on the structure and all the domain boundary $\Gamma$, henceforth taken into account to model phenomena such as reflection, absorption (Le Bot et al., 2000), or more recently diffraction (Reboul et al., 2005). These sources follow the Lambert's law for diffuse reflection which states that the directional distribution of the emitted intensity is proportional to $\cos \theta$,

$$
\sigma_{f}(\mathbf{p}, \mathbf{u})=\sigma_{f}(\mathbf{p}) \cos \theta
$$

where $\theta$ is the angle between the direction of emission and the unit outward normal at point $\mathbf{p}$. Power balance equations on the boundary of the acoustical domain are solved to evaluate the amplitudes of these fictitious sources,

$$
\begin{aligned}
\frac{\sigma_{f}(\mathbf{p})}{4}= & (1-\alpha)\left[\int_{S} \sigma_{e}(\mathbf{q}, \mathbf{u}) \mathbf{H}(\mathbf{q}, \mathbf{p}) \cdot \mathbf{n}_{\mathbf{p}} \mathrm{d} S\right. \\
& \left.+\int_{\Gamma} \sigma_{f}\left(\mathbf{p}^{\prime}, \mathbf{u}^{\prime}\right) \mathbf{H}\left(\mathbf{p}^{\prime}, \mathbf{p}\right) \cdot \mathbf{n}_{\mathbf{p}} \mathrm{d} \Gamma^{\prime}\right]
\end{aligned}
$$


This equation links the amplitude of the fictitious source $\sigma_{f}$ at $\mathbf{p}$ on the domain boundary to the amplitude of the equivalent sources $\sigma_{e}$ at $\mathbf{q}$ on the vibrating structure emitting in the direction $\mathbf{u}$ towards $\mathbf{p}$, and to the amplitude of the fictitious sources $\sigma_{f}$ at $\mathbf{p}^{\prime}$ on $\Gamma$ emitting in the direction $\mathbf{u}^{\prime}$ towards $\mathbf{p}$ (Figure 2 ). $\mathbf{H}(\mathbf{q}, \mathbf{p})=c G(\mathbf{q}, \mathbf{p}) \mathbf{u}$ is the energy kernel for acoustical intensity. $\alpha$ denotes the boundary energy absorption.

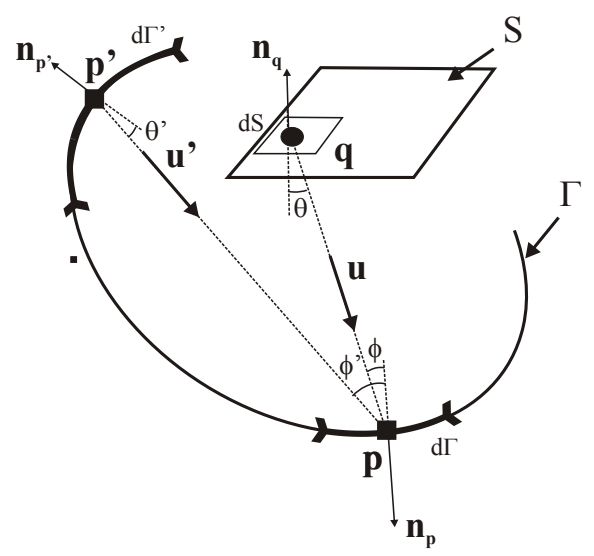

Figure 2. Fictitious sources introduced at $\mathbf{p}$ on the domain boundary $\Gamma$ are linked to the equivalent sources introduced at $\mathbf{q}$ on the vibrating structure $S$ emitting in the direction $\mathbf{u}$ towards $\mathbf{p}$, and to the other fictitious sources at $\mathbf{p}^{\prime}$ on $\Gamma$ emitting in the direction $\mathbf{u}^{\prime}$ towards $\mathbf{p}$

The radiated field $W$ is then given by summing the contributions of all the sources, equivalent and fictitious, so that,

$$
W(\mathbf{r})=\int_{S} \sigma_{e}(\mathbf{q}, \mathbf{u}) G(\mathbf{q}, \mathbf{r}) \mathrm{d} S+\int_{\Gamma} \sigma_{f}(\mathbf{p}, \mathbf{u}) G(\mathbf{p}, \mathbf{r}) \mathrm{d} \Gamma
$$

The radiative transfer method relies on the assumption of uncorrelated sources, valid in the high-frequency range. The mesh is coarser than for standard finite element methods that is a numerical advantage. Besides, computations are carried out on frequency bands which contributes to decrease the computational cost.

\subsection{Numerical implementation}

The Fredholm equation of second order for sources $\sigma$ is solved using the collocation method. The boundary $\Gamma$ is divided into elements denoted $\Gamma_{k}$ where a collocation 
point $\mathbf{p}_{\mathbf{k}}$ is introduced at the barycentre. $\sigma_{f}(\mathbf{p})$ is assumed to be constant on each element and its value is denoted $\sigma_{f, k}$. Thus, Equation [11] to solve is written as :

$$
\begin{aligned}
\frac{\sigma_{f, k}}{4} & =(1-\alpha)\left[\sum_{s} \int_{S} \sigma_{e, s}(\theta) H\left(\mathbf{q}, \mathbf{p}_{\mathbf{k}}\right) \cos \phi \mathrm{d} S\right. \\
& \left.+\sum_{l} \sigma_{f, l} \int_{\Gamma_{l}} \cos \theta^{\prime} H\left(\mathbf{p}^{\prime}, \mathbf{p}_{\mathbf{k}}\right) \cos \phi^{\prime} \mathrm{d} \Gamma^{\prime}\right]
\end{aligned}
$$

where $\theta^{\prime}$ is the emission angle at point $\mathbf{p}^{\prime}, \phi$ is the incidence angle at point $\mathbf{p}_{\mathbf{k}}$ of the acoustical ray coming from $\mathbf{s}$ (first term of the right member) and from $\mathbf{p}^{\prime}$ (second term of the right member).

This equation can be written in matrix form as :

$$
(I d-T) \sigma=g
$$

where,

$$
\begin{aligned}
& T_{k l}=4(1-\alpha) \int_{\Gamma_{l}} \cos \theta^{\prime} H\left(\mathbf{p}^{\prime}, \mathbf{p}_{\mathbf{k}}\right) \cos \phi^{\prime} \mathrm{d} \Gamma^{\prime} \\
& g_{k}=4(1-\alpha) \int_{S} \sigma_{e, s}(\theta) H\left(\mathbf{q}, \mathbf{p}_{\mathbf{k}}\right) \cos \phi \mathrm{d} S .
\end{aligned}
$$

Integrals are solved using a Gaussian quadrature rule. Let us note that the matrix $\mathrm{T}$ is a real, full and non-symmetric matrix. Its inversion is the most time-consuming operation. Once the sources $\sigma_{f, k}$ known, the energy field $W$ is deduced from the discrete form of Equation [12],

$$
W(\mathbf{r})=\sum_{s} \int_{S} \sigma_{e, s}(\theta) G(\mathbf{q}, \mathbf{r}) \mathrm{d} S+\sum_{k} \sigma_{k} \int_{\Gamma_{k}} G(\mathbf{p}, \mathbf{r}) \cos \theta \mathrm{d} \Gamma
$$

\section{Application}

\subsection{Model description}

Let us consider a power plant inside the engine compartment of a truck with absorbent panels as depicted in Figure 3. The power plant is composed with the engine, the clutch housing and the gearbox. Absorbent panels under the engine are intended to reduce the noise radiated outside the compartment. The upper and the lower panels are respectively made with foams of $50 \mathrm{~mm}$ and $25 \mathrm{~mm}$ thick. This study focuses on the noise radiated due to the vibrations of the upper face of the gearbox housing. The gearbox is modelled as a two-stage train of helicoidal gears and the vibrations are induced by an harmonic unit force inside the action plan of the gear mesh and normal to the denture of the entry couple. 


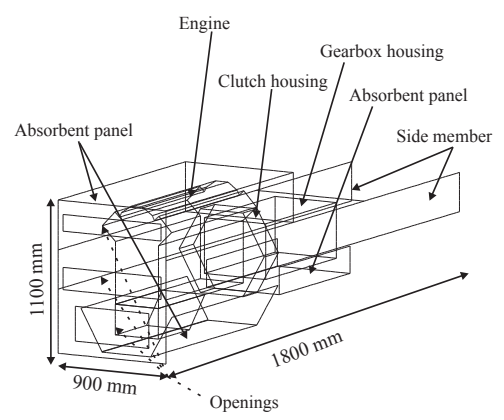

Figure 3. Description of the power plant including the gearbox housing located inside an engine compartment with absorbent panels

\subsection{Acoustical radiation of the upper face of the gearbox housing}

Firstly, the noise radiated in the free-field by the upper face of the gearbox housing is computed using the equivalent sources as defined in Equation [5], [6] and [7]. This comparison is intended to check the validity of the equivalent sources. Results are compared to those obtained using the Boundary Element Method implemented in the commercial software Sysnoise ${ }^{\mathrm{TM}}$. Figures 4 and 5 present the sound pressure level respectively on a double plane surrounding the face, and on a plane perpendicular to this one, computed with the BEM (Figures (a)) and with the equivalent sources (Figures (b)). Maps obtained with the equivalent sources are in good agreement with the BEM ones in terms of amplitude and directivity.

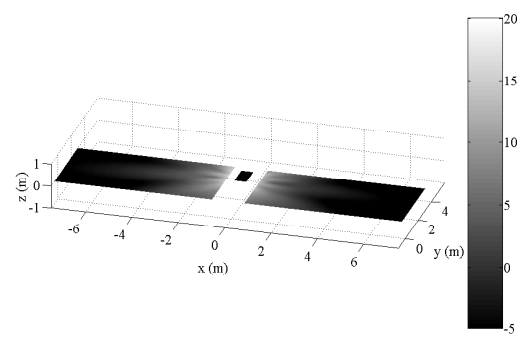

(a)

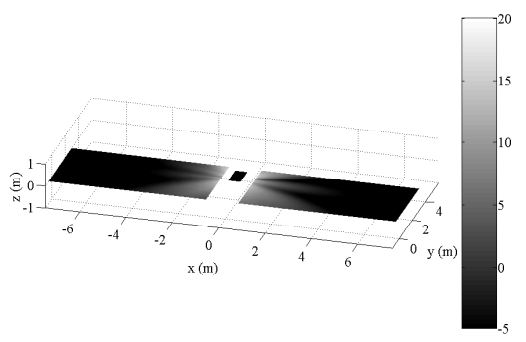

(b)

Figure 4. Sound Pressure Level ( $\mathrm{dB}$ ref : $2 \cdot 10^{-5} \mathrm{~Pa}$ ) radiated in the free-field by the upper face of the gearbox housing on a double plane surrounding the power plant computed with (a) the BEM, (b) the equivalent sources. Computations are carried out on the octave band centred on $1000 \mathrm{~Hz}$ 


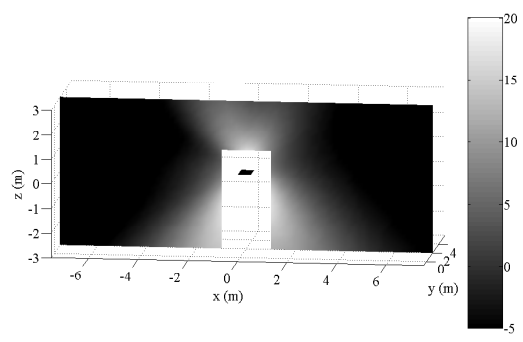

(a)

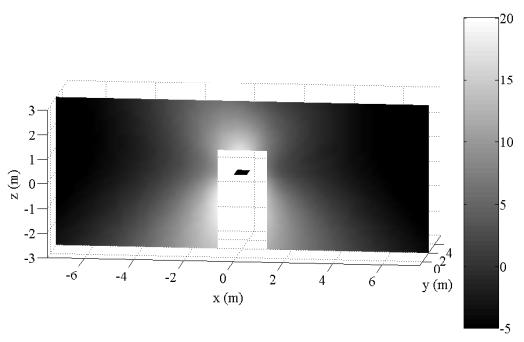

(b)

Figure 5. Sound Pressure Level ( $\mathrm{dB}$ ref : $\left.2 \cdot 10^{-5} \mathrm{~Pa}\right)$ radiated in the free-field by the upper face of the gearbox housing on a plane perpendicular to the power plant computed with (a) the BEM, (b) the equivalent sources. Computations are carried out on the octave band centred on $1000 \mathrm{~Hz}$

\subsection{Acoustical radiation outside the engine compartment}

Once again, results obtained with the hybrid approach are compared to those given by the Boundary Element Method implemented in the commercial software Sysnoise $^{\mathrm{TM}}$. The BEM mesh is shown on Figure 6. It consists of 12769 quadrangular elements and it is valid up to $1420 \mathrm{~Hz}$ according to the criteria of 6 elements per wavelength. BEM calculations are carried out at pure-tone frequency every $50 \mathrm{~Hz}$ between 700 and $1400 \mathrm{~Hz}$. Computation time is about 6 hours on a $2.53 \mathrm{GHz}$ Pentium 4 with RAM 512 Mo. These calculations are then averaged on the third-octave band centred on $1000 \mathrm{~Hz}$. The mesh for the radiative transfer method includes 367 triangular elements and 72 equivalent sources. Computation time is about 1 hour on a $2.4 \mathrm{GHz}$ Pentium 4 with RAM 512 Mo.

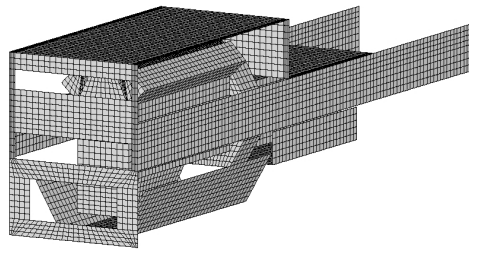

Figure 6. BEM mesh of the whole engine compartment

Figures 7 and 8 present the sound pressure level respectively on a double plane surrounding the compartment, and on a plane perpendicular to this one, computed with the BEM (Figures (a)) and with the hybrid method (Figures (b)). The proposed method allows to reproduce the mean acoustical radiated field, in particular the decrease as the distance from the structure increases. Interferences are not reproduced because of the assumption of uncorrelated sources. The main differences are observed above the absorbent panel on the top of the gearbox. Indeed, contrary to the BEM, the hybrid 
method predicts no field in this area. These differences are assigned to the diffraction phenomena which was not modelled in the radiative transfer method implemented for this study. Indeed, an absorbent panel is located just above the energy sources on the upper face of the gearbox, and so the acoustical field behind this panel is mainly due to diffraction effects. Thus, the numerical implementation of this phenomena in the radiative transfer method is under development based on the theoretical work published in (Reboul, 2005).

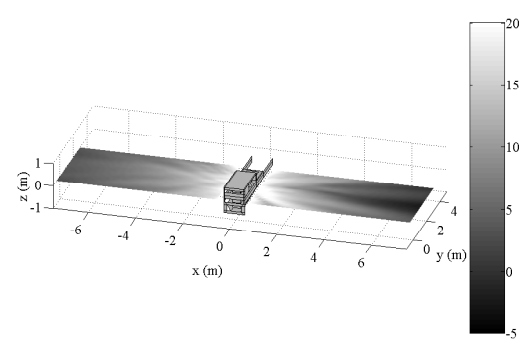

(a)

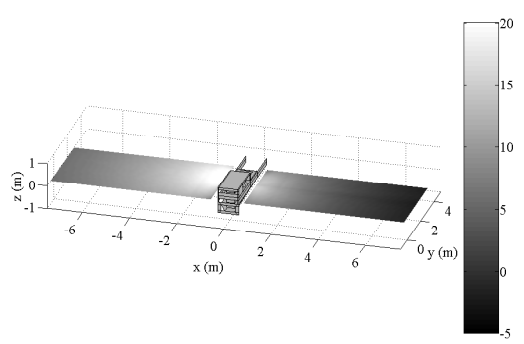

(b)

Figure 7. Sound Pressure Level ( $\mathrm{dB}$ ref : $2 \cdot 10^{-5} \mathrm{~Pa}$ ) radiated by the upper face of the gearbox housing on a double plane surrounding the power plant computed with (a) the BEM, (b) the hybrid method. Computations are carried out on the octave band centred on $1000 \mathrm{~Hz}$

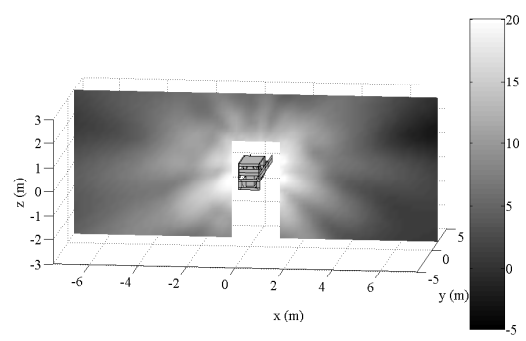

(a)

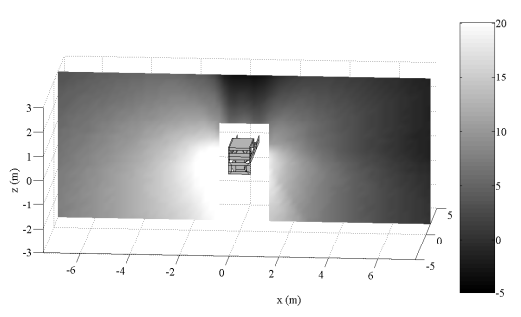

(b)

Figure 8. Sound Pressure Level ( $\mathrm{dB}$ ref : $\left.2 \cdot 10^{-5} \mathrm{~Pa}\right)$ radiated by the upper face of the gearbox housing on a plane perpendicular to the power plant computed with (a) the BEM, (b) the hybrid method. Computations are carried out on the octave band centred on $1000 \mathrm{~Hz}$

\section{Conclusion}

The proposed hybrid method allows to apply the radiative transfer method to predict the noise radiated in the high frequency range by a vibrating structure from the 
knowledge of its vibratory behaviour. The main point of the method is the introduction of equivalent sources for which analytical formulations are given. The study of the noise radiated around an engine compartment has shown that the method is adapted to the prediction of the mean acoustical behaviour around the vibrating structure, interferences are not described because sources are assumed to be uncorrelated. Main interests of the approach are that computational costs are reduced compared to more conventional analysis such as BEM analysis as meshes are coarser and as computations are carried out at the centre frequency of frequency bands.

\section{References}

Guyader J.-L., Loyau T., « The frequency averaged quadratic pressure : a method for calculating the noise emitted by structures and for localising the acoustic sources », Acta Acustica, vol. 86, p. 1021-1027, 2000.

Kim J.-K., Ih J.-G., « Prediction of sound level at high-frequency bands by means of a simplified boundary element method », J. Acoust. Soc. Am., vol. 112, n 6, p. 2645-2655, 2002.

Kuttruff H., « Energetic sound propagation in rooms », Acust. Acta Acust., vol. 83, p. 622-628, 1997.

Le Bot A., Bocquillet A., « Comparison of an integral equation on energy and the ray tracing technique in room acoustics », J. Acoust. Soc. Am., vol. 108, n 4, p. 1732-1740, 2000.

Lesueur C., Rayonnement acoustique des structures - Vibroacoustique, Interactions des Etudes et Recherches d'Electricité de France, Eyrolles, 1988.

Miles R., « Sound field in a rectangular enclosure with diffusely reflecting boundaries », Journal of Sound and Vibration, vol. 92, p. 203-226, 1984.

Reboul E., Vibroacoustique des mécanismes à hautes fréquences - Application aux transmissions par engrenages, Thèse de doctorat, École Centrale de Lyon, 2005.

Reboul E., Le Bot A., Perret-Liaudet J., « Radiative transfer equation for multiple diffraction », J. Acoust. Soc. Am., vol. 118, n 3, p. 1326-1334, 2005.

Sadoulet-Reboul E., Le Bot A., Perret-Liaudet J., Mori M., Houjoh H., « A hybrid method for vibroacoustics based on the radiative energy transfer method », Journal of Sound and Vibration, vol. 303, n 3-5, p. 675-690, 2007.

Wang A., Vlahopoulos N., Wu K., « Development of an energy boundary element formulation for computing high-frequency sound radiation from incoherent intensityboundary conditions », Journal of Sound and Vibration, vol. 278, n 1-2, p. 413-436, 2004. 
